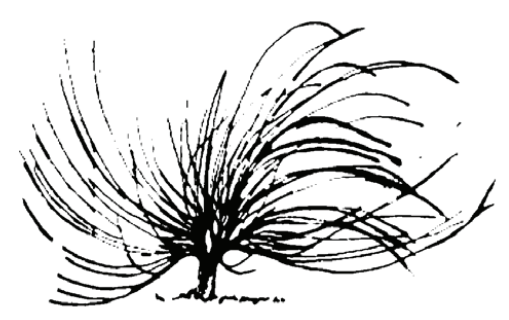

\title{
Orientación del Capital Humano a Través del Enfoque en Competencias Laborales en una Empresa Colombiana
}

\author{
Hugo Alexander Vega Riaño ${ }^{1}$ \\ Universidad de Pamplona \\ Colombia \\ havega@gmail.com
}

Samuel Duarte Figueroa ${ }^{2}$

Universidad de Pamplona

Colombia

samuelduarte@unipamplona.edu.co

\section{Resumen}

Este estudio desarrolla el concepto de capital humano tomado como un símbolo de valor, formando referencias a la importancia que tienen las personas en toda organización. Acá se resalta el precio inigualable que hay en cada uno de los miembros de una empresa, el hombre como centro, el cual cada uno de las partes sistémicas giran en búsqueda de la excelencia. Además, a medida que el capital humano incremente sus conocimientos crece su potencial. Sobre la base de todo capital humano se encuentran las actitudes; en ellas se ven las conductas de las personas y como toda conducta se puede modificar.

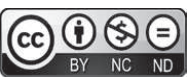

Recibido: 16 de julio de 2015-Aprobado: 4 de diciembre de 2015

1 Profesor Universidad de Pamplona (Colombia). Doctor en Psicología Social y Antropología de las Organizaciones. Universidad de Salamanca (España).

2 Profesor Universidad de Pamplona (Colombia). Magister en Administración de empresas en la Universidad de la Salle. 
Por tanto, es un compromiso muy importante en el caso de un jefe, ya que entra a jugar un papel significativo el mundo interno de los recursos humanos y su relación con sus semejantes. Por tanto, este estudio desarrolla un proceso que oriente el capital humano a empresas colombianas que propongan estrategias y fundamentos para el desarrollo de competencias laborales en empleados y operarios (enfoques tradicionales, enfoques por competencias), esto nos lleva a analizar e identificar las bases generales en las que descansa el manejo del capital humano en la empresa colombiana. Para la realización del estudio se utilizó el enfoque por competencias, articulando las diferentes fuentes y formas de indagación, desde el medio natural de la cotidianidad laboral, partiendo desde la lógica de los actores involucrados, de la práctica laboral y de las estrategias para el manejo del capital humano utilizadas en la empresa colombiana, para llegar a unas estrategias formativas laborales en competencias. La gestión del capital humano teniendo en cuenta las competencias, concebida como una síntesis de práctica y de teoría, es una de las varias opciones del mundo moderno frente a la enorme brecha existente entre la educación formal, escolarizada. Desde esta perspectiva, el manejo del capital humano debe ser concebido como un proceso orientado a lograr que el individuo tenga las capacidades, habilidades, conocimientos, destrezas y experiencias que una empresa exige para ocupar determinado cargo.

Palabras clave: capital humano, empresa, actitudes, conductas, práctica laboral, estrategias formativas laborales, conductas

\begin{abstract}
This study develops the concept of human capital taken as a symbol of value, making references to the importance of people in any organization. Here the great price that is in each one of the members of a company, the man at the center, who each of the parties revolve around in systemic pursuit of excellence are highlighted. Moreover, as the human capital increase their knowledge grows its potential. On the basis of all human capital are the
\end{abstract}


attitudes; in these, people's behaviors can be modified. Therefore, it is a very important commitment in the case of a head as it comes to play a significant role the inner world of human resources and their relationship with others. Therefore, this study develops a process that guides the human capital to Colombian businesses and foundations to propose strategies for skills development for employees and workers (traditional approaches, competency-based approaches); this leads us to analyze and identify the general basis on which rests the management of human capital in the Colombian company. The competency approach was used for the study, coordinating the various sources and forms of inquiry, from the environment to labor daily, starting from the logic of the actors involved, labor practice and management strategies human capital used in the Colombian company to reach a labor competency training strategies. Human capital management taking into account the competencies, conceived as a synthesis of practice and theory, is one of several options the modern world against the huge gap between formal education, schooling. From this perspective, human capital management must be conceived, as a program to ensure that the individual has the skills, abilities, knowledge, skills and experience requires a company to take specific charge process.

Keywords: human capital, enterprise, attitudes, behaviors, job training, job training strategies, behaviors

\section{Formación tradicional}

La formación tradicional se caracterizaba por buscar una capacitación para un puesto de trabajo, teniendo en cuenta ciertos criterios generales acerca de que habilidades o experiencias debía tener el trabajador, pero no se tenía en cuenta el objetivo del cargo, las competencias reales que se requerían en el orden cognitivo, técnico, social y corporativo, para cumplir con dicho objetivo.

"De ahí las constantes señales de alerta acerca de la obsolescencia, tanto de categorías de pensamiento como de aspectos institucionales; la necesidad de idear nuevas formas organizativas y nuevas 
metodologías; la imperiosidad de incorporar el cambio como un dato permanente en cualquier actividad, o la rutina del cambio permanente" (Suárez, 2005, p. 32).

Precisamente porque interesa fijar la atención en las consecuencias e implicancias de las transformaciones ocurridas y en curso, se realiza este breve abordaje al tema de las relaciones entre el Estado y el sector privado en la formación de capital humano, concibiendo la situación actual como un punto en un proceso de cambio, profundamente vinculada a un desarrollo histórico que presente más recurrencias de las que en principio podría pensarse.

Muchas de las formas de acción tradicional del Estado han sido cuestionadas y, específicamente se asiste a un proceso de crítica y revisión de las formas de acción pública en el campo de la formación. Los cuestionamientos y las alternativas que se plantean son, efectivamente, nuevos; no es, en cambio, novedad que los sistemas e instituciones de formación sean escenarios de profundos debates y cuestionamientos.

"Las instituciones de formación, concebidas originalmente como entidades de capacitación no formal, independientes de los sistemas regulares de educación y dotadas de gran autonomía y fluidos lazos con el mercado de trabajo, hicieron su primera aparición a comienzos de los años cuarenta"(Suarez, 2005, p. 42).

Desde entonces, han estado inmersas en los profundos cambios que durante el período ha experimentado el contexto económico, social, político, cultural y tecnológico, poniendo a prueba su capacidad de adaptación y su real validez institucional. Sobrevivieron a sucesivas y distintas crisis, y lograron -con suficiente éxito revalidar su función cada vez que las circunstancias y presiones emergentes de las nuevas situaciones nacionales que se fueron dando, las pusieron en tela de juicio.

"En este contexto, el escenario de la formación y desarrollo de capital humano adquiere una vez más en la historia de los países de América Latina y el Caribe una importancia estratégica. En este ámbito es donde la nueva estrategia de desarrollo, en su meta de lograr una transformación productiva que permita elevar tanto sus niveles de competitividad y productividad como de equidad social, se juega varias de sus cartas principales" (Serrato, 2010. p. 72).

Podría afirmarse, sin error, que no es fortuito que los países que han emprendido con mayor éxito, esfuerzos de transformación productiva y modernización, hayan concedido la mayor importancia al 
desarrollo de su capital humano, lo cual los ha llevado a la «reconversión» de sus programas educativos y de formación y capacitación profesional, bien sea que ellos tengan lugar dentro de las empresas o fuera de ellas (Serrato, 2010).

El hecho es de tal profundidad y significación que, aun los países más avanzados, revisan hoy sus sistemas educativos y de formación, con el fin de elevar su calidad, vincularlos con mayor eficiencia al desarrollo productivo, científico y tecnológico, y encaminarlos como fuerza motriz de una capacidad productiva y competitiva cada día mayor.

\section{Formación por competencias laborales}

Entre los principales cambios que ha traído la afluencia de nuevas concepciones que existen a nivel mundial está la denominada sociedad de la información que reconceptualiza todo lo existente sobre trabajo humano y la denominada sociedad del conocimiento que valoriza todo lo que es y significa trabajo.

De manos de estas dos concepciones: "El trabajo es ahora concebido como el aporte para lograr los objetivos de la organización, por lo que las metodologías actuales de análisis del trabajo en lugar de preocuparse por las tareas que el trabajador debe desempeñar, se orientan a establecer cuál es el aporte que este trabajador hace al logro de los objetivos propuestos por su empresa" (UNAB. El Enfoque de Competencia Laboral 2005, p. 5).

Las empresa de hoy se han tecnificado, han modernizado sus estructuras y reconocen la importancia de contar con el talento humano que se requiere para lograr dichos objetivos. "Existe una concepción importante, debido a la mecanización de las empresas, que es la siguiente: el trabajo pesado, el repetitivo puede ser hecho por máquinas, el creativo, el que genera nuevos productos, el que mejora los existentes es hecho por el hombre y para eso se requiere talento, por eso ha tomado tanto valor el talento humano" (UNAB. El Enfoque de Competencia Laboral 2005).

Ahora ese talento que está destinado al logro de los objetivos de la organización, es utilizado por los empleados y los trabajadores en forma de conocimientos, de destrezas, de habilidades experiencias anteriores y comprensión del proceso productivo para lograr los resultados esperados.

"En la actualidad es importante la formación y es fundamental que exista un espacio amplio de aplicación de dicho conocimiento por 
parte del trabajador, de tal manera, que el incremento de los conocimientos se puede obtener mayores resultados, se obtienen los objetivos propuestos y se incrementan las capacidades con las cuales una empresa puede ser competitiva" (Bárcenas, 2010., p.18).

Igualmente se valora la capacidad de trabajar en equipo, se aboga por el trabajo "decente", en el que se obtienen los ingresos y las condiciones de vida que los trabajadores se merecen y se están diseñando mecanismos tendientes a la valorización y al reconocimiento de las capacidades demostradas en el trabajo.

Otro aspecto fundamental es que el mundo de hoy es: Un mundo competitivo, en el que las empresas lo deben ser y en alto grado, por lo tanto, se ha generado la necesidad de que la empresa trabaje como un equipo competente, por lo que es obvio que debe estar formada por trabajadores competentes (Bárcenas, 2010). Por eso está creciendo la demanda de competencias tales como la capacidad de comprender, procesar y aplicar un gran número de informaciones en el trabajo y las múltiples interacciones que se requieren entre los equipos de trabajo, lo cual ha generado que sean muy valoradas las habilidades para comunicarse rápidamente.

Hoy en Latinoamérica, la formación del trabajador es un instrumento económico para la formación de valor agregado, pero dicha formación no es solo un derecho de los trabajadores, ya que es también un importante instrumento económico que alimenta las políticas activas de empleo y las estrategias de productividad y competitividad de las empresas. Desde esta perspectiva, la formación interesa a gobiernos, empresas y empleadores (Castañeda, 2010).

Paralelamente, el avance tecnológico influye para que la competitividad más rentable y duradera se obtenga a través de la calidad y el valor agregado y no de la disminución del costo. Y es claro igualmente, que calidad y valor agregado requieren también, de mano de obra capacitada y del aprovechamiento pleno de todo capital humano, lo que incluye a varones y mujeres. Por ello, hay una estrecha relación entre la adecuada gestión de personal, el fortalecimiento de la competitividad empresarial, igualdad de trato y de oportunidades en el trabajo.

"Por otro lado, es obvio que la productividad de un trabajador adecuadamente capacitado será muy superior a la de otro no calificado, y solo en la medida que exista dicha preparación podrá participar en comités de Gerencia, comprometiéndose en los de negocios de su empresa 
en torno a una visión empresarial total; coadyuvando en la producción de información en todos los niveles de la organización; compromiso de los diferentes niveles con las cifras de la empresa; participando en forma determinante en el establecimiento y desarrollo de planes de acción e inclinándose por una organización de trabajo más completa" ${ }^{3}$.

Ahora bien. Especialmente para los países subdesarrollados, la ventaja competitiva que significa contar con mano de obra calificada depende, también, de la capacidad del país de retener a las personas que se han formado y de emplearlas apropiadamente en esas actividades. Como puede verse, reaparece aquí un fuerte ligamen con el trabajo. Es menester calificar, que para que esa inversión sea económicamente rentable, se debe garantizar trabajo.

En este último aspecto: "La ventaja es compartida por el trabajador y el empleador. Este dispondrá de un trabajador con iniciativa, polifuncional o con aptitudes para la polifuncionalidad, activo y competitivo, mientras que esas mismas condiciones permitirán al trabajador adaptarse a los cambios y así disponer de mayores posibilidades de conservar su puesto de trabajo"4.

Pero la inversa también es cierta. No solo la formación incide positivamente sobre el trabajo, sino que además este favorece el mejoramiento de la capacitación. En efecto, las competencias se desarrollan en gran medida en el trabajo, por lo cual, un trabajo es un ámbito y una ocasión de aprendizaje, perfeccionamiento y actualización. En este plano: "La modalidad de la formación continua desempeña el rol estelar. Y entonces, así como el acceso a la formación es un requisito del trabajo, también se constata que el trabajo asegura la formación continua. Así aparecen en escena -otra vez- nuevos derechos laborales cuya eficacia viabiliza la formación en el trabajo, en especial, tiempo libre para capacitación"5.

Para entender que es una competencia, conviene discriminar su significado del de otro término, más antiguo y ampliamente utilizado: el de calificación. "Si por calificación se entendió, con alguna frecuencia, el conjunto de conocimientos y habilidades que los individuos adquieren durante los procesos de socialización y educación/formación, se estaría en presencia de un enfoque de carácter instrumental; de ahí que sería una especie de «activo» con que las personas contarían para

3 Ibíd., Pág. 18.

4 Ibíd., Pág. 21.

5 Ibíd., Pág. 21. 
desempeñar determinados puestos. En este sentido, se la definiría como la «capacidad potencial para desempeñar o realizar las tareas correspondientes a una actividad o puesto»" (García, 2010, p.12).

La competencia, por su parte, refiere sólo a algunos aspectos de este acervo de conocimientos y habilidades: aquellos que son necesarios para llegar a ciertos resultados exigidos en una circunstancia determinada. Es la «capacidad real para lograr un objetivo o resultado en un contexto dado».

"Para identificar la calificación requerida en un puesto o bien en el mercado de trabajo, el método que en muchos casos se adaptó fue el de un análisis ocupacional orientado al establecimiento de un simple inventario de todas las tareas que comprendía una ocupación. El inventario de tareas era el punto de referencia" (Solarte, 2010, p.45.)

Para identificar la competencia: "Se parte de los resultados y/u objetivos deseados de la organización en su conjunto, que derivan en tareas y estas en conocimientos, habilidades y destrezas requeridas. En este caso, las tareas son concebidas como un medio cambiante entre el resultado y la dotación de conocimientos y habilidades del individuo. Para evitar interferencias y reduccionismos al definir primero las tareas, se busca establecer una conexión directa entre resultados y dotación de conocimientos y habilidades" 6 .

"En un ambiente de continuos cambios técnicos y de organización en las empresas, las tareas también cambian. Por su parte, los objetivos en general son menos cambiantes, aunque tienden a ser cada vez más exigentes. Tenerlos como punto de referencia para la dotación de conocimientos y habilidades requeridas tendría más sentido que las tareas" (Cabezas, 2009, p.48).

\section{La nueva ciudadanía}

En las últimas décadas de este siglo, asistimos a una serie de procesos que configuran lo que indudablemente puede reconocerse como un cambio de era. La era industrial nacida a la luz de la Revolución Francesa, de la revolución científica y de la revolución industrial, la cual da paso a otra era ¿posmoderna? ¿Posindustrial? Hoy el problema es cómo se construye una opción superadora, cómo se construye la

$6 \quad$ Ibíd., Pág. 22 
nueva democracia. ¿Qué nueva forma debe adoptar hoy "el gobierno del pueblo para el pueblo"? ¿Qué condiciones humanas deben desarrollar, quienes serán los ciudadanos de esta nueva sociedad?

\section{La sociedad del conocimiento}

Un rasgo indudable de esta nueva era es la importancia sin precedentes que adquiere el saber científico tecnológico. Son claras las tendencias que indican que ingresamos en la "era del conocimiento".

"Es el más importante porque es el más humano. Porque sólo conoce (aprende) el ser humano, pero también porque "el conocimiento tiene virtudes intrínsecamente democráticas. A diferencia de las fuentes de poder tradicionales (la fuerza, el dinero, la tierra), el conocimiento es infinitamente ampliable" (Durán, 2010, p.113). Su utilización no lo desgasta sino que, al contrario, puede producir más conocimiento. Un mismo conocimiento, puede ser utilizado por muchas personas y su producción exige creatividad, libertad de circulación, intercambios, críticas constructivas, diálogo.

Una "sociedad del conocimiento" se perfila, entonces, como una forma social superadora de las actuales, a condición de que el conocimiento - que es la base - sea un bien que está disponible para todos." Esta es la nueva sociedad. Mucho conocimiento al alcance de todos, distribuido de tal manera que garantice igualdad de oportunidades". ¿Cómo se logra? A través de un sistema escolar que sea el encargado de garantizarlo, al cual toda la sociedad le dé no solamente el mandato de hacerlo, sino también los recursos para lograrlos.

\section{De las reformas a la transformación}

Como consecuencia de las transformaciones económicas, de los cambios en los modos de producción, de los procesos de globalización de la economía y de la cultura, una respuesta común que aparece en todas las latitudes son las reformas de la educación. Pero los frutos no han sido los esperados. Su común denominador es su dificultad para abrirse paso, cuando no su fracaso. ¿A qué se debe esto? "Las fuertes transformaciones de la sociedad global están dejando en claro que este fin de siglo está suponiendo un cambio total de reglas de juego, un cambio de paradigma. En el caso de la educación, quizás el fracaso se deba a que 
las respuestas tienden a sostener una mirada retrospectiva más que a inventar una propuesta prospectiva" (Cierra, 2010, p.37).

Y esto es grave ya que a pesar de que estas respuestas prospectivas existen en otras áreas, en la educación parece que se prefiriera una respuesta que se acerca a más de lo mismo. Nos limitamos a expandir y replicar el modelo clásico de la escuela del siglo pasado (más bien del siglo ante-pasado, del siglo XVIII, que a esta altura, ya casi es del siglo ante-ante-pasado), modernizándolo con materiales didácticos y, sobre todo, incluyendo la mayor cantidad de computadoras posible. Esta solución es por demás ingenua y denota muy poca capacidad de mirada prospectiva.

"Se requiere un nuevo paradigma educativo que pueda acompañar los nuevos paradigmas que surgen en las demás áreas de la sociedad. Otro paradigma que, superando las restricciones del actual, sea capaz tanto de saldar las deudas del pasado cuanto de dar respuestas más adecuadas a las necesidades del futuro" (Cierra, 2010, p.37).

Lo que se ha hecho habitualmente, y se está haciendo, es tratar de llevar a su máximo rendimiento el modelo de educación conocido en occidente a partir de los comienzos de la era moderna, sin tratar de encontrar una propuesta superadora.

Por esto las estrategias que se llevan a cabo no están siendo suficientes para dar respuestas diferentes para una sociedad que se perfila como diferente, ya que apuestan a mejorar este sistema educativo, nacido de las necesidades de la revolución francesa y de la revolución industrial, hoy superadas. Es necesario situarse fuera de esta óptica, para plantearse un nuevo sistema educativo. Es necesario un cambio de paradigma.

\section{De los saberes a las competencias}

El nuevo paradigma implica cambiar básicamente la ecuación conocimiento a docente a empleado u operario pasando de los saberes a las competencias. "Los elementos que definen la estructura básica del sistema educativo son de diferente orden, pero pueden distinguirse a partir de diferentes niveles de análisis un conjunto de principios vertebradores y estructurantes (formas soportantes) que rigen la organización de sus distintas instancias" (Smills, 2010, p. 72). Los varios ejes subyacentes funcionan como organizadores de la estructura básica de la educación y determinan aspectos específicos de su organización. 
Estos principios vertebradores se agrupan en tres grandes niveles de análisis. Un primer nivel político-ideológico, un segundo nivel técnico-pedagógico y un tercer nivel organizacional. Cada uno de ellos implica al otro. "Es decir, el nivel político-ideológico, que es el que da el 'sentido' al sistema educativo, determina el campo de posibilidades del nivel técnico-pedagógico. A su vez, el técnico-pedagógico, que se refiere a las decisiones estrictamente 'educativas', determina qué forma debe tener la organización concreta de la educación" (Smills, 2010, p. 74).

Las definiciones que se asumen en estos tres niveles de análisis en una formación social determinada, definen un 'paradigma' educativo. Actualmente, está en crisis el paradigma clásico de la educación, o sea, los acuerdos sociales básicos aceptados en la sociedad en relación con las definiciones implicadas en estos tres niveles.

Aunque existen elementos que permiten atisbar algunas redefiniciones, estos no han crecido todavía lo suficiente ni están lo suficientemente sistematizados y organizados, como para plasmarse en decisiones que afecten medularmente a los sistemas educativos. Las decisiones se toman, todavía, desde las miradas retrospectivas del futuro. Frente a la crisis del paradigma clásico no se vislumbran respuestas alternativas. Son propuestas de 'reforma' y no de transformación estructural.

Quizás porque resulta demasiado extraña a nuestro léxico, al campo de la educación no ha llegado todavía la idea de 'reingeniería', es decir, de volver a pensar una organización en todas sus dimensiones para que pueda cumplir mejor su función. En nuestro caso, cumplir mejor la función sería dar educación de mejor calidad, a más cantidad de gente, con menores costos.

"En el paradigma de organización del sistema escolar que hemos heredado, se entiende por 'conocimiento' la actividad humana que tiene como objetivo la descripción y explicación de los fenómenos de la realidad, con el fin de generar teoría que permita predecir su comportamiento. El conocimiento se orienta hacia la producción de teoría, y por lo tanto, el objetivo de la escuela será la adquisición de "saberes" que se definen fundamentalmente desde su dimensión teórica," (Smills, 2010, p. 77).

Un sistema educativo orientado hacia las necesidades del siglo XXI debe incorporar una definición de ciencia (conocimiento) que la entienda con el enfoque de Investigación y Desarrollo, o sea, como la actividad humana que explica los diferentes campos de la realidad y 
genera teoría, tratando de producir cambios en ellos. En este enfoque, el fin fundamental de la ciencia es operar sobre la realidad para transformarla. Este no es un cambio menor, porque supone pasar de una actitud pasiva y contemplativa, a una activa y de intervención sobre la realidad. Es la redefinición que subyace y da razón a la idea de que la escuela no se debe centrar ya sólo en los 'saberes', sino que debe poder dar cuenta de la formación de las 'competencias' para el hacer.

El paradigma de educación que hemos heredado define el 'aprendizaje' como la resultante de un proceso de estímulo-respuesta, en el que tiene un papel preponderante el esfuerzo individual, el estímulo de los textos y la acción de transmisión del docente sobre el empleado u operario. Es decir, la relación docente-empleado u operario debe ser directa, personal y permanente.

"Un sistema educativo orientado hacia las necesidades del siglo XXI, debe entender el aprendizaje como el resultado de la construcción activa del sujeto sobre el objeto de aprendizaje. Supone un aprendiz activo, que desarrolla hipótesis propias acerca de cómo funciona el mundo, que deben ser puestas a prueba permanentemente" (Carrillo, 2010, p.87). Supone la generación de operaciones mentales y procedimientos prácticos que permitan seguir aprendiendo solo, durante el tiempo de vida que está dentro del sistema educativo y también una vez que egresó del sistema educativo formal. Supone también que el maestro y el empleado u operario exploran y aprenden juntos, pero que esta exploración y aprendizaje mutuo puede revestir diferentes formas, desde las más presenciales hasta las más remotas.

Finalmente, en el paradigma clásico de organización del sistema escolar se supone que lo que se debe enseñar, los 'contenidos', son ciertos elementos conceptuales de las disciplinas, que llegan a ser muchas veces sólo los datos descriptivos singulares (fechas, datos, nombres).

"Las opciones que se toman en torno a qué definición de conocimiento, de aprendizaje y de contenido es válida para una sociedad, determinan desde los planes de estudio hasta la forma organizativa y las prácticas cotidianas concretas del sistema educativo: qué se enseña y en qué contexto organizacional se enseña" (Carrillo, 2010, p.87). Por esto una transformación educativa, para ser estructural, debe proponer cambios fuertes en estos tres aspectos y no atender única o básicamente, a uno de ellos sin ocuparse de manera sustantiva de los demás. 


\section{Competencias}

Los siete 'lenguajes de la modernidad' definen los nuevos 'contenidos' de la educación. Lo que hay que aprender; lo que hay que enseñar. El problema es que ya no alcanza solo con modificar los programas de estudio. Para competir en la sociedad del conocimiento, no basta con saber las reglas de ortografía, el teorema de Pitágoras o las fechas fundamentales de la historia. Ni siquiera basta con escribir bien, manejar las operaciones matemáticas y conocer los procesos históricos.

"La sociedad del siglo XXI requiere de algo más complejo que los meros 'saberes' o conocimientos. Requiere COMPETENCIAS. El viejo paradigma centraba sus esfuerzos en que los chicos 'supieran'. Una buena escuela era aquella capaz de garantizar conocimientos a todos sus empleado u operarios. Hoy pedimos a la escuela no solo saberes, sino 'competencias"' (Torres, 2010, p. 32).

¿Qué son las competencias? Una competencia es un 'saber hacer', con 'saber' y con 'conciencia". El término 'competencia' hace referencia a un conjunto de propiedades de cada uno de nosotros que se están modificando permanentemente y que tienen que someterse a la prueba de la resolución de problemas concretos, ya sea en la vida diaria o en situaciones de trabajo que encierran cierta incertidumbre y cierta complejidad técnica. La gran diferencia es que la competencia no proviene solamente de la aprobación de un curriculum (plan de estudios), sino de la aplicación de conocimientos en circunstancias prácticas. Los conocimientos necesarios para poder resolver problemas no se pueden transmitir mecánicamente; son una mezcla de conocimientos tecnológicos previos y de la experiencia que se consigue con la práctica, muchas veces conseguida en los lugares de trabajo.

Las competencias están en el medio entre los 'saberes' y las 'habilidades'. Entonces, las competencias, como conjunto de propiedades inestables de las personas, que deben someterse a prueba permanentemente, se oponen a las calificaciones que se medían por un diploma y por la antigüedad en la tarea. Lo importante hoy es SER COMPETEN$\mathrm{TE}$, que quiere decir saber hacer cosas, resolver situaciones. Pero como las situaciones son cada vez más complejas, ser competente requiere por un lado de muchos saberes, teóricos y prácticos, y por otro de mucha imaginación y creatividad. 
Por esto no alcanza con reformas que cambien los programas de estudio que, como todos sabemos, es absolutamente imperativo, y se está haciendo; también hay que cambiar los enfoques de las disciplinas que se enseñan, incluir nuevos campos disciplinarios (como tecnología, inglés o computación), y cambiar las metodologías de enseñanza. Pasar de los saberes a las competencias como meta de la enseñanza es realmente importante no solamente porque en una democracia todos tienen derecho al conocimiento válido, sino también porque la buena formación de todos los ciudadanos es hoy el mejor pasaporte hacia una sociedad productiva e integrada socialmente.

\section{Los nuevos empleos y la formación en competencias}

"El siglo XXI es el siglo de la ciencia y de la tecnología. Más ciencia y tecnología en nuestra vida cotidiana (en los aparatos domésticos, en los medicamentos, en las comunicaciones, en los entretenimientos) no ha significado, sin embargo, más ciencia y tecnología en la institución que prepara a las generaciones jóvenes para insertarse en la sociedad, o sea, en la escuela" (Torres, 2010, p. 35).

Esto no fue grave mientras nuestro aparato productivo tampoco. Las naciones afrontan una competencia mundial cada vez más directa e inmediata y su competitividad se reconsidera hoy desde el punto de vista de la competitividad de la fuerza de trabajo. "Según Richard Reich se perfilan para el siglo XXI, tres amplias categorías de trabajo, que corresponden a las tres diferentes posiciones en que estarán las personas. Las llama servicios rutinarios de producción, servicios personales y servicios simbólico-analíticos" (Torres, 2010, p. 37).

Los servicios rutinarios de producción son una etapa en una secuencia. Aunque eran la base de la línea de producción clásica, no han dejado de existir. Un buen ejemplo son los 'data entry' (ingresadores de datos) en la industria de la informática. Los servicios personales son los que se proporcionan de persona a persona, como los vendedores o los peluqueros. Como los anteriores, deben ser competentes en su tarea pero, además, tienen que ser afables, corteses y serviciales. Los servicios simbólico-analíticos, incluyen las actividades expertas y suponen la capacidad de identificar y resolver problemas. Son los que realmente agregan valor y suponen alta calidad, originalidad, destreza y oportunidad. El trabajo en equipo es, por lo general, un aspecto crítico. 
"Los analistas simbólicos hacen de intermediarios, identifican y resuelven problemas valiéndose de símbolos. Para ello, utilizan instrumentos de análisis variados como algoritmos matemáticos, argumentos legales, tácticas financieras, principios científicos, observaciones psicológicas acerca de cómo persuadir o entretener, métodos inductivos o deductivos, o cualquier otro tipo de técnica para resolver problemas (Zampudio, 2010, p. 72).

Lo más importante es que cuanto mayor sea la proporción de analistas simbólicos que exista en la fuerza de trabajo, más valor agregado existe. Esto depende de la cantidad y calidad de los puestos de trabajo que se generen, pero mucho más de cuáles son las competencias generales que tiene la población. En otras palabras, por más que crezca la oferta de trabajo que requiera competencias de analista simbólico, si no existen en la población, no será posible llenarlos.

Finalmente, hay que tener en cuenta que las transformaciones socioeconómicas ocurridas en las últimas décadas obligan a redefinir la relación entre educación y trabajo. "Las 'nuevas' empresas requieren 'nuevos' trabajadores...Hay fáciles acuerdos acerca de qué caracteriza a esos nuevos trabajadores: una sólida formación básica el dominio de algunas aptitudes. Ya no su conocimiento o su información, ya que esta se devalúa rápidamente y ya no tiene sentido económico transmitirla" (Zampudio, 2010, p. 75). Así como la educación no tiene como misión generar empleo sino hacer emplearle a la gente, tampoco la educación tiene ya como misión formar para un puesto de trabajo específico. La vieja definición de las escuelas vocacionales o técnicas tiene que ser reemplazada por otra más amplia en la que el compromiso sea igual al del resto del sistema educativo: en paralelo con las competencias generales que todos tienen que tener, dar las competencias correspondientes a un campo amplio del mundo del trabajo.

\section{Proceso metodológico}

Los objetivos propuestos en este estudio fueron ubicar a la compañía, en un nivel de organización tal que se consiga la certificación ISO 9000 antes del 2015; -buscar mecanismos que propicien en todo el personal el conocimiento, entendimiento e interpretación de la norma ISO 9000 para que las utilicen integralmente como una rutina de nuestra labor, a cumplir en el 2018; -elevar en un 10\% la productividad de la compañía para la conquista de más mercados, en el año 2015; -definir 
proveedores certificados IS0 9000 con productos entregados a tiempo y cumplimiento absoluto de los estándares de calidad, meta para el 2015; y disminuir en un $20 \%$ el número de reclamos por parte de los clientes, durante el 2015.

La presente investigación es de enfoque cualitativo ya que permite hacer una aproximación global de las situaciones laborales para explorarlas, describirlas y comprenderlas de manera inductiva, a partir del enfoque por competencias. Esto permitió articular las diferentes fuentes y formas de indagación, desde el medio natural de la cotidianidad laboral, partiendo desde la lógica de los actores involucrados, esto es, se parte de la práctica laboral y de las estrategias para el manejo del recurso humano utilizadas en la empresa $\mathrm{EMS}^{7}$, para llegar a unas estrategias formativas laborales en competencias.

\section{Proceso metodologico}

\section{Técnicas de recolección}

Se recopiló información de carácter secundario e información de carácter primario. Para la recolección de la información secundaria, se analizaron textos, documentos, manuales y módulos de formación para el desarrollo de competencias en la formación laboral integral, entre otros.

Para la recolección de información secundaria, se realizó una investigación exploratoria que se desarrolló en la empresa EMS de la ciudad de Bucaramanga, permitiendo conocer la forma de pensar, sentir $\mathrm{y}$ actuar de los directivos frente a un proceso de desarrollo laboral por competencias; así mismo por su carácter flexible; las técnicas escogidas para dicha investigación fueron: observaciones, diálogos informales y análisis de documentos de dicha entidad.

\section{Procesamiento de la información}

La orientacion del capital humano a través del enfoque en competencias laborales en una empresa colombiana es un estudio que describe la metodología implementada para el desarrollo de la investigación, el

7 Es una unidad económica del sector metalmecánico que fue seleccionada para conocer sus aspectos generales, su misión, visión y objetivos, para establecer cómo se manejan ciertos procesos de gestión humana. 
análisis de los datos y las etapas propias del proceso investigativo. Se define dentro de este acápite el nivel de investigación del estudio, el tipo de investigación en el cual se enmarcan los objetivos trazados, la población y muestra del estudio y el método de investigación siguiendo las recomendaciones y aportes de Hernández, R; Fernández, C y Baptista, P. (2006) entre otros autores.

El diseño de la investigación comprende las etapas o fases del estudio, es decir, el proceso que sigue el investigador para alcanzar los objetivos propuestos. En la literatura, se hace referencia a diferentes etapas y fases que estructuran la investigación cualitativa. Para este estudio, se consideran los aportes de Rodríguez, G., Gil, J. y García, E. (1996), quienes señalan que la investigación cualitativa sigue por lo general un conjunto de fases a saber: preparatoria, trabajo de campo, analítica e informativa.

La observación participante y las técnicas de registro (para consignar notas base) fueron los instrumentos de recolección de información en ese estudio, se reconstruyeron las escenas observadas; reconstruir la vivencia del investigador y las interpretaciones en el proceso, en lo que respecta a las estrategias utilizadas en los enfoques de manejo del recurso humano por parte de la empresa y el ámbito para un enfoque por competencias.

\section{Fase preparatoria}

En la fase preparatoria, se concreta el proyecto de investigación con el desarrollo de dos etapas: reflexiva y diseño. Al final de esta fase, se concreta su marco teórico-conceptual y las actividades que realizará para la recolección de los datos, el análisis de la información y la interpretación de los resultados.

En la primera etapa (reflexiva), se reúne la literatura relacionada con el tema (orientación del capital humano a través del enfoque en competencias laborales en una empresa colombiana). Con estas acciones previas, el investigador se ubica dentro del amplio espectro del conocimiento producido para definir los elementos teóricos y metodológicos utilizados en otros escenarios de estudio. 
La segunda fase de la investigación corresponde al trabajo de campo. En esta fase, se hizo aplicación de las técnicas antes descritas, se tuvo acceso a la población y se recolectaron los datos a través de las técnicas. La primera etapa de esta fase fue de acceso al campo a fin de reconocer y explorar la población objeto de estudio así como el contexto escolar, lo cual permitió una mejor planeación para la aplicación de las técnicas e instrumentos. Con posterioridad, se hizo la recogida productiva de datos. En esta etapa, se buscó que la aplicación de las técnicas (observación participante y las técnicas de registro) se hiciera de la manera más apropiada a fin de asegurar el mayor grado de objetividad.

\section{Fase analítica}

La fase analítica corresponde a la manera en que se sistematizaron los datos recolectados y el análisis respectivo de cada uno de ellos. La información de la observación participante y las técnicas de registro fueron tabuladas y analizadas e interpretadas. De este modo, cada elemento relevante de las técnicas de recolección de información se sistematizó a fin de interpretarse a la luz del marco teórico-conceptual que sustenta la investigación.

\section{Validez}

Las técnicas de análisis de documentos utilizadas como fueron la recopilación de información secundaria y la observación participante, permitieron triangular la información obtenida en la empresa para verificar la validez interna de los resultados obtenidos a través de estas técnicas, al igual que la de los informantes y así poder ponderar la evidencia. Para la interpretación se relacionaron las teorías establecidas, después del proceso del análisis, con la visión del investigador y la teoría formal sobre competencias que se tomó como base teórica.

\section{Interpretación de la información}

Para la implantación del análisis funcional en la empresa, deben tenerse en cuenta lo siguiente: 
El proceso de selección de empleados y operarios en la empresa se debe iniciar con la identificación de las competencias establecidas y proseguir con la evaluación del candidato frente a dichas competencias, estableciendo de esta forma su idoneidad para la ocupación a la que aspira. De este modo, el proceso de selección se apoyó en las competencias seleccionadas para la empresa, conforme con sus características institucionales o de un área o cargo en particular.

Las competencias generadas de esta forma, facilitaron un marco de criterios por medio de los cuales se llevó a cabo la selección y el resultado de introducir algunas variaciones en las características tradicionales del proceso podrían resumirse en: el cambio de énfasis en la búsqueda de un candidato para un puesto; considerar la diferencia entre competencias personales y competencias técnicas que se requieren para dichos empleados u operarios; e introducir ejercicios de simulación para detectar la posesión de ciertas competencias por los candidatos a dichos cargos.

Normalmente estas competencias se evaluarán en el proceso de selección mediante la aplicación de pruebas de conocimiento y/o ejercicios de aplicación práctica en el trabajo. En todo caso, para poder realizar un proceso de selección basado en competencias, la empresa debe hacer explícito un modelo de gestión por competencias, basado en las habilidades, conocimientos y experiencia que aspira encontrar en un empleado u operario.

Es evidente que las técnicas propias del cargo de empleado $\mathrm{u}$ operario, entendidas estas como las pericias necesarias para el desarrollo de una actividad específica, son el punto de partida, ya que permitirán desarrollar las labores prácticas, operativas y de ejecución propias de determinado desempeño en la empresa.

Una vez que termine el proceso de selección, la flexibilidad y capacidad de adaptación son claves, porque es necesario que como empleados u operarios, quienes sean contratados, se adapten a las exigencias, características y condiciones de la empresa, encasillándose en determinadas funciones, que pueden desarrollarse mediante cambios constantes de actividades, rotación en cargos y funciones.

\section{Resultados del estudio}

De acuerdo con los instrumentos utilizados en este estudio, el resultado de este análisis devela que la orientación del capital humano 
a través del enfoque por competencias laborales en una empresa colombiana parte de la necesidad de valorar no sólo el conjunto de los conocimientos apropiados (saber) y las habilidades y destrezas (saber hacer) desarrolladas por una persona, acá se aprecia la habilidad de responder y enfrentarse a sus propias necesidades. Esto quiere decir, que se enfrente a una aptitud positiva y valorativa (saber ser) que incide sobre los resultados de la acción, por tanto, la tabla 1 sintetiza los resultados de esta investigación.

\begin{tabular}{|l|l|}
\hline \multicolumn{2}{|c|}{ Tabla 1. COMPETENCIAS LABORALES GENERALES } \\
\hline Intelectuales & $\begin{array}{l}\text { Se observo aptitudes intelectuales que de alguna manera } \\
\text { conectan a la atención, la memoria, la concentración, } \\
\text { la solución de problemas, la toma de decisiones y la } \\
\text { creatividad. }\end{array}$ \\
\hline Personales & $\begin{array}{l}\text { En algunos registros y testimonios se evidencia la forma } \\
\text { de actuar adecuada y asertiva en un espacio productivo, } \\
\text { aportando sus talentos y desarrollando sus potenciales, } \\
\text { de las personas. Podríamos decir que la ética y la } \\
\text { inteligencia emocional son conductas que se adaptan al } \\
\text { cambio. }\end{array}$ \\
\hline Interpersonales & $\begin{array}{l}\text { Se observo un alto porcentaje en la capacidad de } \\
\text { adaptación, trabajo en equipo, resolución de conflictos, } \\
\text { liderazgo y buenas relaciones interpersonales en un } \\
\text { espacio productivo. }\end{array}$ \\
\hline Organizacionales & $\begin{array}{l}\text { El análisis de los instrumentos utilizados develan } \\
\text { que: es objetiva la capacidad para gestionar recursos, } \\
\text { orientación e información a lo que podríamos llamar } \\
\text { servicio y aprendizaje a través recomendaciones de } \\
\text { prácticas de otros, este proceso es positivo porque ayuda } \\
\text { al crecimiento humano de la empresa. }\end{array}$ \\
\hline Tecnológicas & $\begin{array}{l}\text { En cuanto a este proceso, se observa la voluntad para } \\
\text { transformar e innovar el conocimiento (procesos, } \\
\text { procedimientos y métodos) y para encontrar soluciones } \\
\text { prácticas. Este proceso contiene competencias } \\
\text { informáticas y la capacidad de identificar, adaptar, } \\
\text { apropiary transferir tecnologías. }\end{array}$ \\
\hline
\end{tabular}




\section{CONCLUSIONES}

El enfoque por competencias es una alternativa de manejo del capital humano, que debe constituirse en pilar básico de la empresa colombiana, ya que permite contar con el personal que posee las habilidades, destrezas, conocimientos y experiencia que requiere, de acuerdo con las características del cargo y las necesidades específicas de una empresa.

Desde esta perspectiva, el desarrollar este enfoque significa, para una empresa como EMS, que su capital humano está capacitado plenamente para el ejercicio de su cargo, sin mayores entrenamientos o capacitaciones previas que implique costo en dinero, tiempo y gestión. No obstante, ello no implica que el empleado u operario no se capacite o entrene, ya que estas variables son factores fundamentales en el enfoque por competencias. Implica que esta capacitación o entrenamiento se convierta en valor agregado y no en el cubrimiento de una deficiencia en sus competencias.

La empresa EMS como entidad económica se preocupa por su desarrollo productivo y la búsqueda de mercados eficientes, pero está caracterizada por una gestión de personal funcionalista que limita su desarrollo y su crecimiento porque no permite la incorporación de la persona que realmente requiere la empresa, generando ineficiencia, sobrecostos en entrenamiento y capacitación y largos períodos de adaptación. Con un manejo de personal basado en competencias, esta empresa puede generar una mayor eficacia y eficiencia laboral y contar con un personal idóneo desde su selección. La vieja escuela de "formar su personal", no es en la actualidad, económica, laboral y corporativamente factible y justificable.

En la empresa EMS se llevan a cabo los procesos de selección de personal, capacitación, evaluación del desempeño y compensación, utilizando las metodologías tradicionales de valoración de actividades y medición de las correspondientes habilidades, selección de acuerdo con parámetros preestablecidos con base en principios universales y desarrollo de planes y programas de capacitación generales y es necesario que acompañando el enfoque propuesto, se implemente en dichas empresas la metodología de análisis funcional de competencias para que los procesos anteriormente mencionados se diseñen teniendo en cuenta los propósitos de la empresa, las actividades y su desagregación y las competencias necesarias para cada una de ellas, con el fin de 
que cuenten con un mecanismo funcional que le permita aprovechar al máximo su personal y desarrollar las habilidades, destrezas y experiencias que son necesarias para cada unidad de competencia.

Desde esta perspectiva, es necesario reestructurar todos los procesos de gestión humana en la empresa EMS, tales como selección, inducción, compensaciones, capacitación y evaluación del personal utilizando el enfoque de competencias y dirigiendo la organización hacia la identificación de dichas competencias como un fundamento para su mayor desarrollo y crecimiento.

Es necesario que la empresa EMS deje los viejos esquemas y se adentre en la nueva metodología de identificación de competencias para lograr un desarrollo más pleno, ajustado a su misión, visión y objetivos, por lo que la presente guía representa un pequeño esfuerzo para ir generando dicho cambio.

\section{Referencias Bibliográficas}

Bárcenas, E. (2010). Formación y Trabajo. Ediciones La Luz. Buenos Aires.

Cabezas, F. (2009). La Formación para el Trabajo en el Siglo XXI. Editora Cinco. Madrid.

Carrillo, J. (2010). Educación y Formación en Colombia. Ediciones Lumen. Bogotá.

Castañeda, L. (2010). Desarrollo del Capital Humano en América Latina. Editora el Dorsel. Caracas.

CINTERFOR/OIT. Boletín técnico interamericano de formación profesional. Competencias Laborales en la Formación Profesional. Montevideo, n.149, mayo-agosto 2000.

CINTERFOR/OIT. Formación y trabajo: de ayer para mañana. Montevideo, 1996. - Cinterfor/OIT. Formación y trabajo decente. Montevideo, 2000.

Durán, A. (2010). La Sociedad del Conocimiento: Reflexiones y Práctica. Editora Centauro. Caracas.

Ermida U. (2001). Trabajo decente y formación profesional. Montevideo: Cinterfor/OIT.

García, E. (2010). Competencias y trabajo: Procesos y funcionalidad. Ediciones Belgrano. Buenos Aires. 
Hernández, R., Fernández, C. y Baptista, P. (2006): Metodología de la investigación. México: McGraw-Hill.

Leibowicz, J. (2000). Ante el imperativo del aprendizaje permanente, estrategias de formación continúa. Montevideo: Cinterfor/ OIT.

Manual para Diseñar Estructuras Curriculares y Módulos de Formación para el Desarrollo de Competencias en la Formación Profesional Integral". Octubre de 2002.

Marin, W. (2010). Análisis Funcional y Competencias. Cuadernos. Ediciones Alvarado. Lima.

Peña, E. (2010). Las competencias laborales, análisis y desarrollo. Cuadernos. Ediciones Sócrates. Santiago de Chile.

Serrato, E. (2010). Capital humano y Formación. Editora La Fuente. Barcelona, España.

Sierra, Dolores. La Educación: Base del desarrollo y el crecimiento. Editora Belmira. Buenos Aires. 2010.

Smills, G. (2010). Educación en Competencias. Prentice- Hall. México.

Solarte, G. (2010). La Formación, dilema para el Desarrollo. Ediciones Consuegra. Caracas.

Suarez, E. (2005). Cuadernos y folios. Ediciones Latinas. Santa Fe de Bogotá.

Sánchez, J. (2001). Aprendizaje visible, Tecnología invisible. Santiago de Chile: Ediciones Dolmen.

Tamayo, M. (2005). El Proceso de la Investigación Cientifica: incluye evaluación y administración de proyectos de investigación. México: Limusa.

Torres, E. (2010). Desarrollo de los procesos de Formación en Competencias. Editora Sumen. Buenos Aires.

UNAB. El Enfoque de Competencia Laboral. Cuadernos, Bucaramanga. 2005.

Zampudio, E. (2003). Un panorama sobre las competencias a nivel mundial. Editorial Temis. Santa Fe de Bogotá.

Zampudio, E (2010). Nuevas metodologías para la Formación Laboral. Ediciones La Espada. Madrid.

Zarifian, P. (2000). El modelo de competencia y los sistemas productivos. Montevideo: Cinterfor/OIT. 